\title{
Catalpol suppresses proliferation and facilitates apoptosis of MCF-7 breast cancer cells through upregulating microRNA-146a and downregulating matrix metalloproteinase-16 expression
}

\author{
CHUAN LIU ${ }^{1}$, FAN WU ${ }^{2}$, YUANWEI LIU ${ }^{3}$ and CONG MENG ${ }^{3}$ \\ ${ }^{1}$ Department of Clinical Medicine, Yantai School of Binzhou Medical University, Yantai, Shandong 264000; \\ ${ }^{2}$ Department of Pediatrics, Dongying Hospital of Shandong Provincial Hospital, Dongying, Shandong 257091; \\ ${ }^{3}$ Center for Interventional Medicine, The Huangdao Branch of Affiliated Hospital of Qingdao University, \\ Qingdao, Shandong 266500, P.R. China
}

Received October 29, 2014; Accepted July 10, 2015

DOI: $10.3892 / \mathrm{mmr} .2015 .4361$

\begin{abstract}
Breast cancer is the most common malignancy in women, and was the second highest cause of mortality in women in 2013. Matrix metalloproteinase-16 (MMP-16) is an enhancer of the invasion of breast cancer cells. The expression of microRNA-146a (miR-146a) has been reported to be significantly greater in patients with breast cancer compared with healthy controls. Catalpol is one of the main active ingredients of Rehmannia, of which the key pharmacological effects are antitumoral and antiproliferative. The present study was performed to investigate the effect of catalpol on breast cancer and to explore the potential therapeutic mechanisms. Cell proliferation was investigated using an MTT assay, and caspase-3 activity assays and flow cytometry were used to assess apoptosis in MCF-7 breast cancer cells. The expression levels of MMP-16 and miR-146a were investigated using gelatin zymography assays and reverse transcription-quantitative polymerase chain reaction, respectively. In addition, MCF-7 cells were transfected with miR-146a and anti-miR-146a to further investigate the effects of catalpol. The results demonstrated that catalpol reduced proliferation and promoted apoptosis in MCF-7 cells, reducing MMP-16 activity and increasing the expression of miR-146a in MCF-7 cells. This suggests that miR-146a may regulate and control the expression levels of MMP-16 in MCF-7 cells. In conclusion, catalpol suppresses proliferation and facilitates apoptosis of MCF-7 breast cancer
\end{abstract}

Correspondence to: Dr Cong Meng, Center for Interventional Medicine, The Huangdao Branch of Affiliated Hospital of Qingdao University, 1677 Wutai Mountain Road, Qingdao, Shandong 266500, P.R. China

E-mail: congmengmr@163.com

Key words: catalpol, breast cancer, proliferation, apoptosis, microRNA-146a, matrix metalloproteinase-16 cells through upregulating miR-146a and downregulating MMP-16 expression.

\section{Introduction}

Breast cancer is the most common malignancy in women worldwide presenting as a key factor affecting women's health, and ranks second (to lung cancer) in the rate of mortality of women in 2013 (1). With the accelerating urbanization of China, breast cancer incidence and mortality are increasing $(2,3)$. The development of comprehensive treatments has led to improved therapeutic efficacy in breast cancer, however there remains a considerable number of patients experiencing recurrence or resistance to conventional treatment (4).

Matrix metalloproteinases (MMPs) are zinc dependent neutral proteases, which exhibit combined actions to degrade all extracellular and basement membrane components (5). The activity regulation of MMP serves an important role in the process of tissue reconstruction and inflammation, and in the growth, invasion and metastasis of tumors (1). Previous studies demonstrated that MMP-2 and -9 are overexpressed in a variety of solid malignant tumors, potentially promoting tumor invasion, metastasis and angiogenesis through the disruption of the basement membrane and the extracellular matrix (6). In addition, studies have demonstrated that levels of MMP-2 and 9 are correlated with the proliferation of tumor cells and apoptosis of endothelial cells (7,8). MMP-15, -16 and -17 are membrane-type MMPs and are able to degrade the ECM and activate MMP-2 and -13 (4,5). Additionally, MMP-16 has been demonstrated to be an enhancer of breast cancer cell invasiveness $(9,10)$.

MicroRNAs (miRNAs) are a class of non-coding single-stranded RNA molecules with a length of approximately 22 nucleotides, which exist widely in eukaryotic cells. miRNAs bind to specific mRNA molecules to inhibit protein translation or regulate protein expression, with certain varieties able to degrade mRNA (11). At present, approximately 500 miRNAs have been identified in the human genome, and 
200 miRNA sequences have been identified to be associated with cancer (12). Specific alterations in miRNA expression are able to initiate and promote the occurrence of cancer, and certain miRNAs may affect oncogenes and tumor suppressor genes $(13,14)$. Expression of microRNA-146 has been demonstrated to reduce metastatic potential in breast cancer cells through the downregulation of nuclear factor $\kappa \mathrm{B}(15)$. Furthermore, the expression levels of miRNA-146a (miR-146a) are significantly higher in patients with breast cancer, when compared with healthy controls (16).

Catalpol is one of the key active ingredients of Rehmannia, which has effects on the nervous and cardiovascular systems, in addition to exhibiting antihyperglycemic, antitumor and antiproliferative activities (17-19). However, previous studies have only demonstrated that catalpol is able to reproduce the diuretic, laxative and hypoglycemic effects of Rehmannia (20). The present study aimed to investigate whether catalpol inhibited cancer growth by upregulating microRNA-146a and downregulating MMP-16 expression.

\section{Materials and methods}

Chemicals and reagents. Catalpol (purity $\geq 98 \%$ ) was obtained from Sigma-Aldrich (St. Louis, MO, USA) and the chemical structure is presented in Fig. 1. Dulbecco's modified Eagle's medium (DMEM) and fetal calf serum (FCS) were obtained from Gibco Life Technologies (Carlsbad, CA, USA). 3-3-(4,5-dimethylthiazol-2-yl)-2,5-diphenyltetrazolium bromide (MTT) and the Caspase-3 Colorimetric Assay kit were purchased from Sangon Biotech Co., Ltd. (Shanghai, China). The Annexin V-Propidium Iodide (PI) Apoptosis Detection kit was obtained from BestBio (Shanghai, China). TRIzol was obtained from Tiandz, Inc. (Beijing, China). TaqMan ${ }^{\circledR}$, the ABI 7900 Real-Time Polymerase Chain Reaction (PCR) machine (Applied Biosystems Life Technologies, Foster City, CA, USA) and SYBR ${ }^{\circledR}$ Green kit were obtained from Qiagen, Inc. (Valencia, CA, USA).

Cell culture. The human breast cancer MCF-7 cell line was purchased from the Animal Experiment Center of Binzhou Medical University (Yantai, China) and cultured with DMEM containing $10 \%$ FCS with penicillin $(100 \mathrm{U} / \mathrm{ml})$ and streptomycin (100 U/ml), which were both purchased from Sigma-Aldrich. The cells were maintained in a humidified chamber at $37^{\circ} \mathrm{C}$ in $5 \% \mathrm{CO}_{2}$ and culture media was renewed every 2-3 days.

MTT assay. Following culture of MCF-7 cells with catalpol ( 0 , 25,50 and $100 \mu \mathrm{g} / \mathrm{ml}$ ) (21) for $0,24,48$ and $72 \mathrm{~h}$, the viability of MCF-7 cells was determined using the MTT assay. MTT $(\sim 10 \mu 1,5 \mathrm{mg} / \mathrm{ml}$; Sangon Biotech Co., Ltd.) was added to each well and cells were incubated for $4 \mathrm{~h}$ in a humidified chamber at $37{ }^{\circ} \mathrm{C}$ in $5 \% \mathrm{CO}_{2}$. Subsequently, the culture medium was removed and $150 \mu \mathrm{l}$ dimethyl sulfoxide (Invitrogen Life Technologies, Carlsbad, CA, USA) was added to each well, which were then agitated for $20 \mathrm{~min}$ at room temperature. Cell viability was determined using an ELISA reader (Thermo Fisher Scientific, Waltham, MA, USA) at $570 \mathrm{~nm}$.

Caspase-3 activity assays. Following culture of MCF-7 cells with catalpol $(0,25,50$ and $100 \mu \mathrm{g} / \mathrm{ml})$ for $48 \mathrm{~h}$, the activity of caspase-3 was measured using the Caspase-3 Colorimetric Assay kit (Sangon Biotech Co., Ltd.). The level of caspase-3 activity was measured at $405 \mathrm{~nm}$ with the ELISA reader.

Flow cytometry. Following culture of MCF-7 cells with catalpol $(0,25,50$ and $100 \mu \mathrm{g} / \mathrm{ml})$ for $48 \mathrm{~h}$, apoptosis was measured using the Annexin V-PI Apoptosis Detection kit according to the manufacturer's instructions (BestBio) and the fluorescence intensity was detected using flow cytometry (Beckman Coulter, Inc., Brea, CA, USA).

Gelatin zymography assays of MMP-16. The MMP-16 level in MCF-7 cells was measured by gelatin zymography. Samples $(\sim 20 \mu \mathrm{l})$ were collected and placed into new centrifuge tubes. Equal volumes $(50 \mu 1)$ of vitreous samples were mixed with sodium dodecyl sulfate (SDS) sample buffer (Invitrogen Life Technologies). The miscible liquids were run through 10\% SDS-PAGE gels (Invitrogen Life Technologies) polymerized with $1 \mathrm{mg} / \mathrm{ml}$ gelatin. Subsequent to electrophoresis, the gel was washed three times for $20 \mathrm{~min}$ at room temperature in $2.5 \%$ Triton X-100 (Invitrogen Life Technologies) to remove SDS, and incubated in a reaction buffer (Invitrogen Life Technologies) at $37^{\circ} \mathrm{C}$ for $12 \mathrm{~h}$. Following incubation, the gel was stained with $0.05 \%$ Coomassie ${ }^{\circledR}$ Brilliant Blue R-250 (Amresco, LLC, Solon, OH, USA) followed by destaining with washing buffer (45\% methanol, $10 \%$ acetic acid) until the bands were clear. MMP-16 was quantified through densitometer measurement using an image-analysis system (Image Lab ${ }^{\text {TM }}$ software; Bio-Rad Laboratories, Inc., Hercules, CA, USA).

Reverse transcription-quantitative PCR analysis of miR-146a expression. Total RNA was extracted from MCF-7 cell samples using TRIzol according to the manufacturer's protocol. cDNAs were synthesized using TaqMan ${ }^{\circledR}$ and the ABI 7900 Real-Time PCR machine, according to the manufacturer's instructions. The expression of miR-146b was detected with the SYBR ${ }^{\circledR}$ Green kit according to the manufacturer's instructions. The primer sequences were as follows: miR-146a, forward 5'-CTA GCTAGCGGCCGCTAGTAACCCATGGAATTCAGTTCT CAG-3' and reverse 5'-TCGACTGAGAACTGAATTCCA TGGGTTACTAGCGGCCGCTAG-3'; U6, forward 5'-TGA CTTCAACAGCGACACCCA-3' and reverse 5'-CACCCT GTTGCTGTAGCCAAA-3'. The cycling conditions were as follows: $95^{\circ} \mathrm{C}$ for $60 \mathrm{sec}, 40$ cycles of $95^{\circ} \mathrm{C}$ for $30 \mathrm{sec}$ and $60^{\circ} \mathrm{C}$ for $45 \mathrm{sec}$, then $72^{\circ} \mathrm{C}$ for $30 \mathrm{sec}$. The U6 gene was used as an internal control to normalize variances.

Transfection of miR-146a and anti-miR-146a. miR-146a and anti-miR-146a plasmids were designed by and purchased from BioSune Biotechnology Co,Ltd.(Shanghai, China). The plasmids were transfected into MCF-7 cells using Lipofectamine 2000 (Invitrogen Life Technologies) according to the manufacturer's instructions. Following transfection for $24 \mathrm{~h}, \mathrm{MCF}-7$ cells were treated with catalpol $(50 \mu \mathrm{g} / \mathrm{ml})$ for $48 \mathrm{~h}$.

Statistical analysis. The data were analyzed with SPSS software, version 17.0 (SPSS, Inc., Chicago, IL, USA) in the current study. To compare the two groups, Student's t-test was used. $\mathrm{P}<0.05$ was considered to indicate a statistically significant difference. 


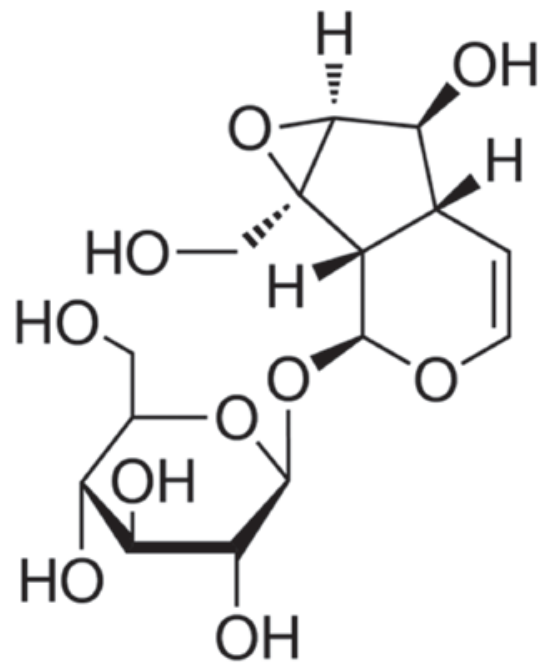

Figure 1. The chemical structure of catalpol.

A
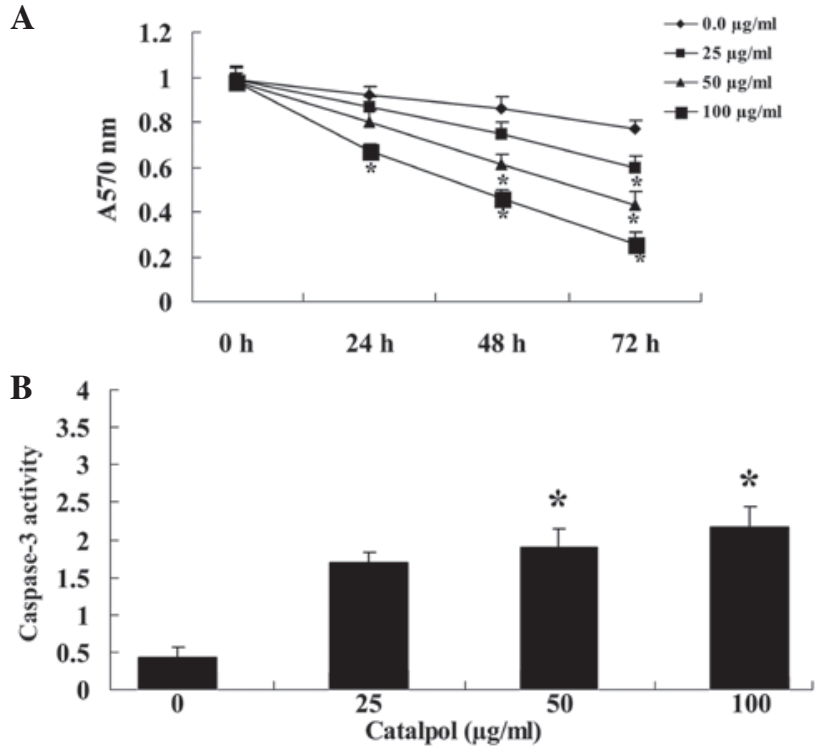

Figure 2. MTT analysis and caspase-3 activity measurement. (A) Catalpol treatment inhibited MCF-7 cell growth. (B) Activity of caspase-3 was significantly increased following treatment. ${ }^{*} \mathrm{P}<0.01$ vs. the $0 \mu \mathrm{g} / \mathrm{ml}$ catalpol treatment group.

\section{Results}

MTT analysis and the activity of caspase-3. An MTT assay was used to determine whether the antiproliferative effect of catalpol $(0,25,50$ and $100 \mu \mathrm{g} / \mathrm{ml})$ reduced the proliferation of MCF-7 cells. The effect of catalpol was dose-dependent, as increasing concentrations of catalpol further reduced the proliferation of MCF-7 cells (Fig. 2A). In addition, the effect of catalpol was time-dependent (Fig. 2A). Following treatment with catalpol (50 and $100 \mu \mathrm{g} / \mathrm{ml}$ ) for $48 \mathrm{~h}$, the activity of caspase-3 in MCF-7 cells was significantly increased $(\mathrm{P}<0.01$; Fig. 2B).

Flow-cytometric analysis for the detection of apoptosis. To determine whether MCF-7 cells undergo apoptosis upon treatment with catalpol $(0,25,50$ and $100 \mu \mathrm{g} / \mathrm{ml})$ for $48 \mathrm{~h}$,

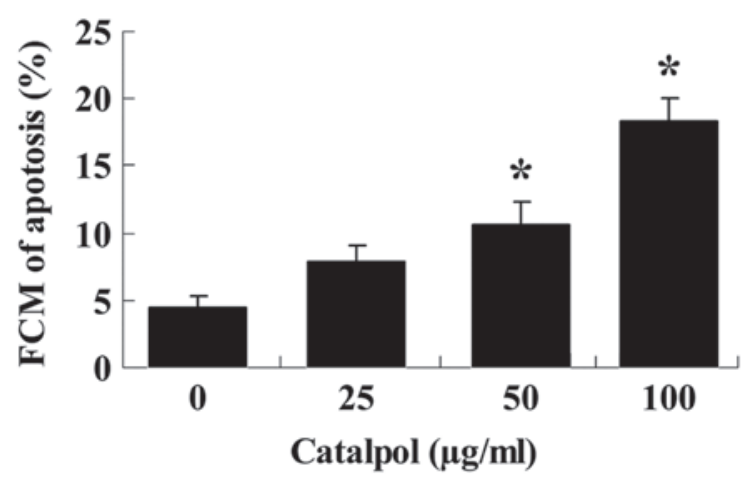

Figure 3. Flow-cytometric analysis for cellular apoptosis. Catalpol significantly increased apoptosis of MCF-7 cells following $48 \mathrm{~h}$ treatment. ${ }^{*} \mathrm{P}<0.01$ vs. the $0 \mu \mathrm{g} / \mathrm{ml}$ catalpol treatment group. FCM, flow cytometry.

A

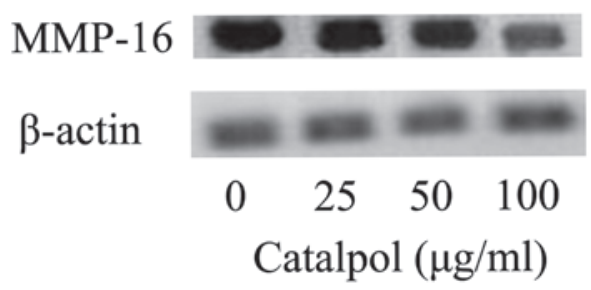

B

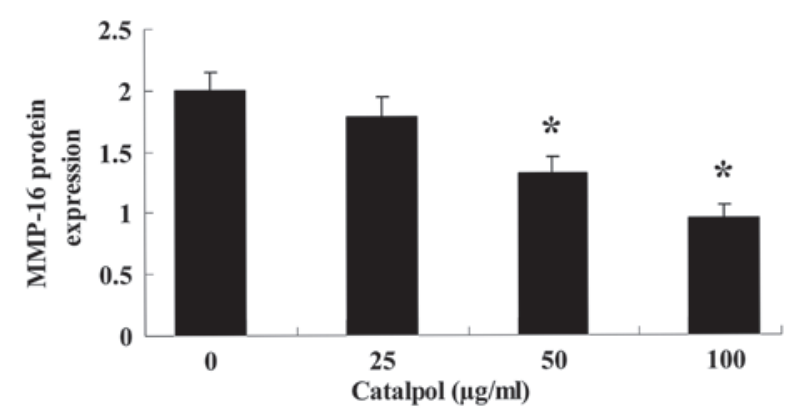

Figure 4. Inhibition effect of catalpol on MMP-16. (A) MMP-16 activity was reduced in a dose-dependent manner in MCF-7 cells following treatment with catalpol as measured by gelatin zymography. (B) Quantification of MMP-16 protein level. ${ }^{*} \mathrm{P}<0.01$ vs. the $0 \mu \mathrm{g} / \mathrm{ml}$ catalpol treatment group. MMP-16, matrix metalloproteinase-16.

annexin V-IP was used to measure the apoptotic cells. The addition of catalpol $(0,25,50$ and $100 \mu \mathrm{g} / \mathrm{ml})$ for $48 \mathrm{~h}$ induced concentration-dependent apoptosis of MCF-7 cells (Fig. 3). The percentage of apoptotic MCF-7 cells in the 50 and $100 \mu \mathrm{g} / \mathrm{ml}$ catalpol groups was significantly increased ( $\mathrm{P}<0.01$; Fig. 3$)$.

Catalpol inhibits expression of MMP-16. To investigate the effect of catalpol upon MMP-16, a gelatin zymography assay was used to analyze the MMP-16 protein levels in MCF-7 cells (Fig. 4). When treated with catalpol (50 and $100 \mu \mathrm{g} / \mathrm{ml}$ ) for $48 \mathrm{~h}$, the MMP-16 protein levels in MCF-7 cells were significantly reduced $(\mathrm{P}<0.01$; Fig. 4$)$.

Catalpol increases miR-146a expression. As expression of miR-146a had been previously observed in MCF-7 cells (22), its expression was investigated following treatment with 


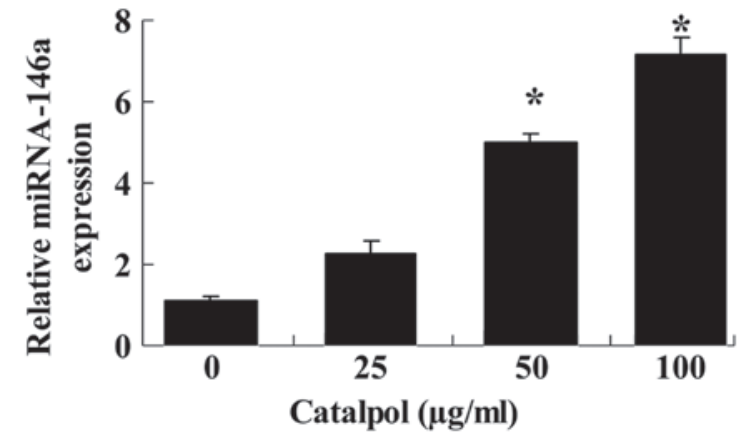

Figure 5. Catalpol activates miR-146a expression. Catalpol treatment increased the expression of miR-146a dose-dependently. ${ }^{*} \mathrm{P}<0.01$ vs. the $0 \mu \mathrm{g} / \mathrm{ml}$ catalpol treatment group. miR-146a, microRNA-146a.

A

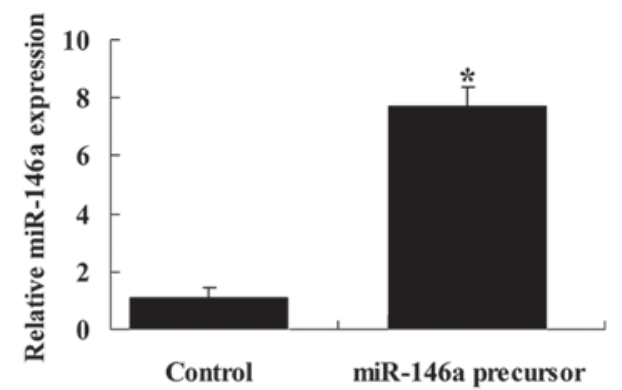

B

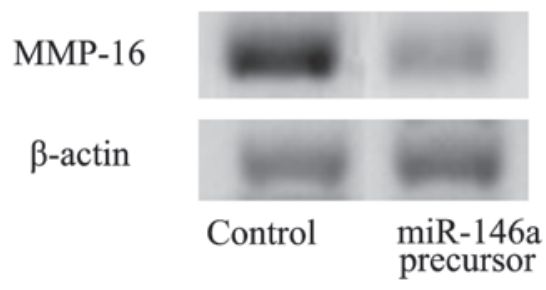

Figure 6. Overexpression of miR-146a and MMP-16 expression. (A) miR-146a plasmids significantly increased the expression of miR-146a. (B) Transfection with miR-146a reduced MMP-16 expression. ${ }^{*} \mathrm{P}<0.01$ vs. the control group. miR-146a, microRNA-146a; MMP-16, matrix metalloproteinase-16.

catalpol. The expression of miR-146a in MCF-7 cells was demonstrated to increase following treatment with catalpol $(0,25,50$ and $100 \mu \mathrm{g} / \mathrm{ml})$ for $48 \mathrm{~h}$ (Fig. 5). Treatment with 50 and $100 \mu \mathrm{g} / \mathrm{ml}$ catalpol resulted in a significant increase in the level of miR-146a expression in MCF-7 cells $(\mathrm{P}<0.01$; Fig. 5).

Overexpression of miR-146a and MMP-16 expression. To further investigate the role of miR-146a expression in MCF-7 cells, cells were transfected with miR-146a. The results demonstrated that overexpression of miR-146a significantly increased the expression of miR-146a in MCF-7 cells $(\mathrm{P}<0.01$; Fig. 6A). In addition, overexpression of miR-146a also reduced the expression of MMP-16 in MCF-7 cells (Fig. 6B).

Anti-miR-146a is able to reverse the effect of catalpol. To further investigate the association between catalpol and miR-146a expression, anti-miR-146a was transfected into MCF-7 cells that were subsequently treated with catalpol. Transfection of MCF-7 cells with anti-miR-146a resulted in a significant reduction in miR-146a expression ( $\mathrm{P}<0.01$; Fig. 7A).
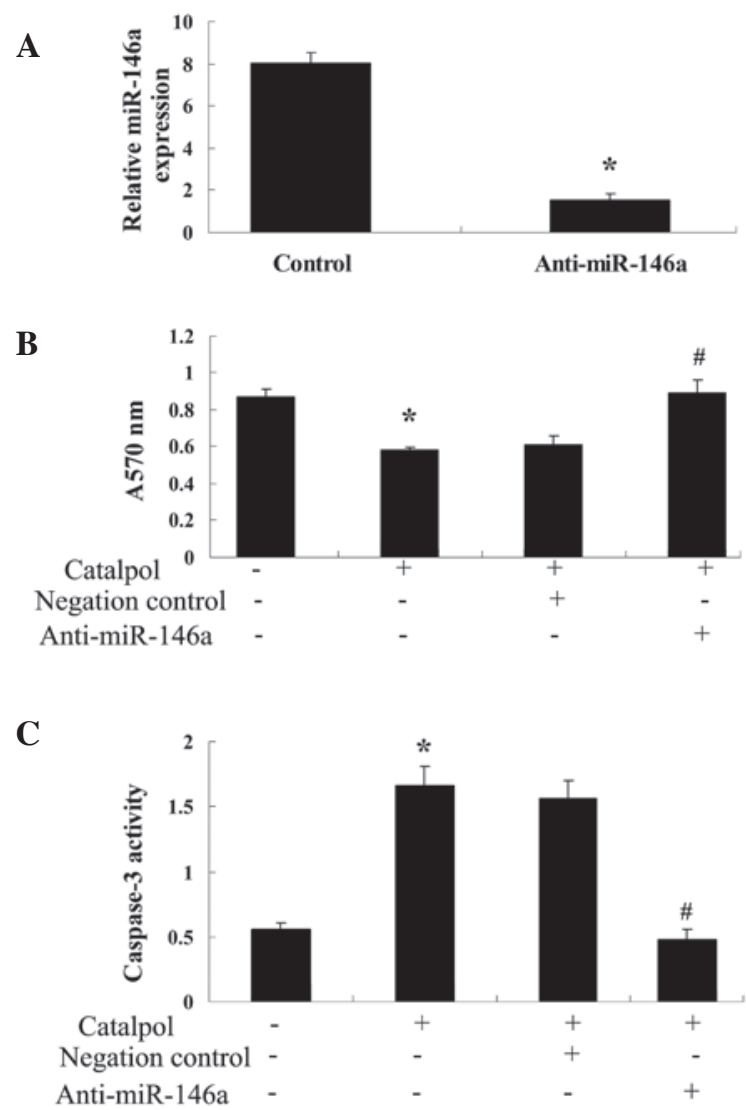

D

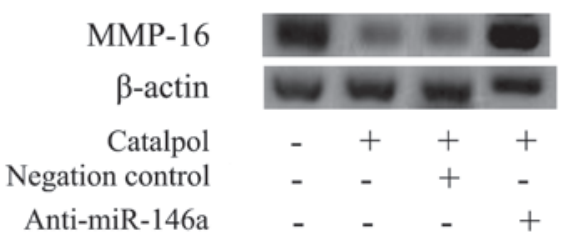

Figure 7. Anti-miR-146a is able to reverse the effect of catalpol. (A) Anti-miR-146a significantly reduced the expression of miR-146a in MCF-7 cells. (B) Following treatment with catalpol $(50 \mu \mathrm{g} / \mathrm{ml})$ for $48 \mathrm{~h}$, anti-miR-146a increased cellular proliferation. (C) Following treatment with catalpol $(50 \mu \mathrm{g} / \mathrm{ml})$ for $48 \mathrm{~h}$, anti-miR-146a reduced apoptosis of MCF-7 cells. (D) Anti-miR-146a significantly increased MMP-16 activity in MCF-7 cells following catalpol $(50 \mu \mathrm{g} / \mathrm{ml})$ treatment at $48 \mathrm{~h}$. ${ }^{*} \mathrm{P}<0.01 \mathrm{vs}$. the $0 \mu \mathrm{g} / \mathrm{ml}$ catalpol treatment group, ${ }^{\#} \mathrm{P}<0.01$ vs. the catalpol-treated group transfected with negative control. miR-146a, microRNA-146a; MMP-16, matrix metalloproteinase-16.

Following treatment with $50 \mu \mathrm{g} / \mathrm{ml}$ catalpol, expression of anti-miR-146a significantly reduced the effect of catalpol on cellular proliferation $(\mathrm{P}<0.01$; Fig. 7B) and apoptosis in MCF-7 cells $(\mathrm{P}<0.01$; Fig. 7C). Furthermore, anti-miR-146a prevented the reduction in MMP-16 levels following catalpol treatment of MCF-7 cells ( $\mathrm{P}<0.01$; Fig. 7D).

\section{Discussion}

Breast cancer is the most common malignancy in women in numerous countries and regions, presenting a serious threat to women's health (9). In China, a trend towards rapid growth in incidence rates has been observed. In the present study, the effect of catalpol was demonstrated to reduce the 
proliferation of MCF-7 cells in a time- and dose-dependent manner. Previously, it was observed that catalpol is able to inhibit the A2780 sensitive ovarian cancer cell line (23). The present study demonstrated that catalpol is additionally able to promote apoptosis and increase the activity of caspase- 3 in MCF-7 cells. Wang et al (24) reported that catalpol attenuated ischemia-induced apoptotic death via suppressing the activation of caspase-3. Liang et al (25) observed that treatment with catalpol attenuated neuronal apoptosis also through regulating the activity of caspase-3 and -9 .

The growth, invasion and metastasis of tumor cells is a complex process with multiple steps and stages. MMPs are involved in the process by which tumor cells degrade the basement membrane, infiltrate into the surrounding matrix to induce angiogenesis, approach adjacent lymphatic vessels, penetrate endothelial cells of the basement membrane and form secondary tumors (26). Different tumor types express different MMPs, and the degree to which breast cancer is malignant is positively correlated with the overexpression of MMP-2 and -9 (27). In the present study, it was demonstrated that treatment with catalpol was able to reduce the level of MMP-16 protein expression in MCF-7 cells. Previous studies indicate that catalpol possesses activity against human epidermoid carcinoma, human rhabdomyosarcoma, transgenic murine L-cells cancer cell lines and African green monkey kidney cells $(28,29)$.

Previous studies have demonstrated that miRNA is involved in the occurrence and development of tumors by targeting important tumor-associated genes (30-32). miR-101, $-129,-130,-133 \mathrm{a}$ and -144 have been reported to potentially inhibit the migration of MDA-MB-231 to various degrees (33). miR-106b-25 gene clusters have been demonstrated to promote drug resistance in breast cancer cells, and the effect is not achieved by altering the expression of P-gp (34). Sandhu et al (35) reported that miR-146a resulted in reduced proliferation and increased apoptosis of breast cancer cells and Wang et al (36) indicated that miR-146a expression suppresses CXCR4-mediated human breast cancer migration. The present study suggested that treatment with catalpol may effectively increase the miR-146a expression in MCF-7 cells. Notably, the upregulation of expression of miR-146a may control the expression of MMP-16 in MCF-7 cells. In addition, downregulation of miR-146a expression was observed to induce MMP-16 expression in MCF-7 cells. Therefore, miR-146a may regulate and control the expression of MMP-16 levels in MCF-7 cells. In support of this, the present study demonstrated that downregulation of expression of miR-146a reduced the effect of catalpol on cellular proliferation and apoptosis of MCF-7 cells.

In conclusion, the current study demonstrated the effects of catalpol on cellular proliferation and cell death in breast cancer cells. Catalpol treatment resulted in the upregulation of miR-146a expression and downregulation of MMP-16 expression in MCF-7 cells. Furthermore, downregulation of miR-146a expression resulted in an upregulation of the expression levels of MMP-16 in catalpol-treated MCF-7 cells. Taken together, these results suggest that catalpol may be therapeutically beneficial in breast cancer through the upregulation of miR-146a expression and the downregulation of MMP-16 expression.

\section{References}

1. Hu XC, Zhang J, Xu BH, et al: Cisplatin plus gemcitabine versus paclitaxel plus gemcitabine as first-line therapy for metastatic triple-negative breast cancer (CBCSG006): A randomised, openlabel, multicentre, phase 3 trial. Lancet Oncol 16: 436-446, 2015

2. Fu JF, Chen HL, Yang J, Yi CH and Zheng S: Feasibility and accuracy of sentinel lymph node biopsy in clinically node-positive breast cancer after neoadjuvant chemotherapy: A meta-analysis. PLoS One 9: e105316, 2014.

3. Nie XC, Dong DS, Bai Y and Xia P: Meta-analysis of black tea consumption and breast cancer risk: Update 2013. Nutr Cancer 66: 1009-1014, 2014.

4. Noh EM, Chung EY, Youn HJ, Jung SH, Hur H, Lee YR and Kim JS: Cis-guggulsterone inhibits the IKK/NF- $\kappa \mathrm{B}$ pathway, whereas trans-guggulsterone inhibits MAPK/AP-1 in MCF-7 breast cancer cells: Guggulsterone regulates MMP-9 expression in an isomer-specific manner. Int J Mol Med 31: 393-399, 2013.

5. Cai KQ, Yang WL, Capo-Chichi CD, Vanderveer L, Wu H, Godwin AK and Xu XX: Prominent expression of metalloproteinases in early stages of ovarian tumorigenesis. Mol Carcinog 46: 130-143, 2007.

6. Fan SH, Wang YY, Lu J, et al: CERS2 Suppresses Tumor Cell Invasion and Is Associated with Decreased V-ATPase and MMP-2/MMP-9 activities in Breast Cancer. J Cell Biochem 116: 502-513, 2015.

7. Xie M, Hu A, Luo Y, Sun W, Hu X and Tang S: Interleukin-4 and melatonin ameliorate high glucose and interleukin-lbeta stimulated inflammatory reaction in human retinal endothelial cells and retinal pigment epithelial cells. Mol Vis 20: 921-928, 2014.

8. Tang C, Chen L, Gu W, et al: Cyclosporin A enhances the ability of trophoblasts to displace the activated human umbilical vein endothelial cell monolayers. Int J Clin Exp Pathol 6: 2441-2450, 2013.

9. Delassus GS, Cho H, Park J and Eliceiri GL: New pathway links from cancer-progression determinants to gene expression of matrix metalloproteinases in breast cancer cells. J Cell Physiol 217: 739-744, 2008.

10. Hegedüs L, Cho H, Xie X and Eliceiri GL: Additional MDA-MB-231 breast cancer cell matrix metalloproteinases promote invasiveness. J Cell Physiol 216: 480-485, 2008.

11. Lee RC, Feinbaum RL and Ambros V: The C. elegans heterochronic gene lin-4 encodes small RNAs with antisense complementarity to lin-14. Cell 75: 843-854, 1993.

12. Hede K: Studies define role of microRNA in cancer. J Natl Cancer Inst 97: 1114-1115, 2005.

13. Croce CM and Calin GA: miRNAs, cancer, and stem cell division. Cell 122: 6-7, 2005.

14. Gregory RI and Shiekhattar R: MicroRNA biogenesis and cancer. Cancer Res 65: 3509-3512, 2005.

15. Bhaumik D, Scott GK, Schokrpur S, Patil CK, Campisi J and Benz CC: Expression of microRNA-146 suppresses NF-kappaB activity with reduction of metastatic potential in breast cancer cells. Oncogene 27: 5643-5647, 2008.

16. Kumar S, Keerthana R, Pazhanimuthu A and Perumal P: Overexpression of circulating miRNA-21 and miRNA-146a in plasma samples of breast cancer patients. Indian J Biochem Biophys 50: 210-214, 2013.

17. Xiao WQ, Yin GJ, Fan YT, Qiu L, Cang XF, Yu G, Hu YL, Xing M, Wu Q, Wang XP, et al: Catalpol ameliorates sodium taurocholate-induced acute pancreatitis in rats via inhibiting activation of nuclear factor kappa B. Int J Mol Sci 15: 11957-11972, 2014.

18. Huang WJ, Niu HS, Lin MH, Cheng JT and Hsu FL: Antihyperglycemic effect of catalpol in streptozotocin-induced diabetic rats. J Nat Prod 73: 1170-1172, 2010.

19. Lü J, Wang Y, Zhao W, Li N, Li H, Lu J, Zeng W, Bao S and Bai Y: Effects of catalpol, L-shikonin and paeonol extracted from radix rehmanniae, radix arnebiae and cortex moutan on KGF-induced $\mathrm{HaCaT}$ cell proliferation. Zhonghua Yi Xue Za Zhi 94: 1265-1269, 2014 (In Chinese).

20. García C, León LG, Pungitore CR, Ríos-Luci C, Daranas AH, Montero JC, Pandiella A, Tonn CE, Martín VS and Padrón JM: Enhancement of antiproliferative activity by molecular simplification of catalpol. Bioorg Med Chem 18: 2515-2523, 2010.

21. Chen C, Chen Z, Xu F, Zhu C, Fang F, Shu S, Li M and Ling C: Radio-protective effect of catalpol in cultured cells and mice. J Radiat Res (Tokyo) 54: 76-82, 2013. 
22. Pogribny IP, Filkowski JN, Tryndyak VP, Golubov A, Shpyleva SI and Kovalchuk O: Alterations of microRNAs and their targets are associated with acquired resistance of MCF-7 breast cancer cells to cisplatin. Int J Cancer 127: 1785-1794, 2010.

23. Pungitore CR, León LG, García C, Martín VS, Tonn CE and Padrón JM: Novel antiproliferative analogs of the Taq DNA polymerase inhibitor catalpol. Bioorg Med Chem Lett 17: 1332-1335, 2007.

24. Wang Z, An LJ, Duan YL, Li YC and Jiang B: Catalpol protects rat pheochromocytoma cells against oxygen and glucose deprivation-induced injury. Neurol Res 30: 106-112, 2008.

25. Liang JH, Du J, Xu LD, Jiang T, Hao S, Bi J and Jiang B: Catalpol protects primary cultured cortical neurons induced by Abeta(1-42) through a mitochondrial-dependent caspase pathway. Neurochem Int 55: 741-746, 2009.

26. Loo WT, Chen JP, Chow LW and Chou JW: Effects of Shugansanjie Tang on matrix metalloproteinases 1,3 and 9 and telomerase reverse transcriptase expression in human breast cells in vitro. Biomed Pharmacother 61: 601-605, 2007.

27. Fagan-Solis KD, Schneider SS, Pentecost BT, Bentley BA, Otis CN Gierthy JF and Arcaro KF: The RhoA pathway mediates MMP-2 and MMP-9-independent invasive behavior in a triple-negative breast cancer cell line. J Cell Biochem 114: 1385-1394, 2013.

28. Liu YF, Zhao Y, Wen XS and Dong QT: Advances in research on pharmacodynamics and chemical conversion of catalpol. Zhongguo Zhong Yao Za Zhi 32: 1128-1130, 2007 (In Chinese).
29. Saracoglu I and Harput US: In vitro cytotoxic activity and structure activity relationships of iridoid glucosides derived from Veronica species. Phytother Res 26: 148-152, 2012.

30. Adams BD, Kasinski AL and Slack FJ: Aberrant regulation and function of microRNAs in cancer. Curr Biol 24: R762-R776, 2014.

31. Maxwell GL, Shoji Y, Darcy K, et al: MicroRNAs in endometrial cancers from black and white patients. Am J Obstet Gynecol 212: e191-e110, 2015

32. Seven M, Karatas OF, Duz MB and Ozen M: The role of miRNAs in cancer: from pathogenesis to therapeutic implications. Future Oncol 10: 1027-1048, 2014.

33. D'Ippolito E and Iorio MV: MicroRNAs and triple negative breast cancer. Int J Mol Sci 14: 22202-22220, 2013.

34. Zhou Y, Hu Y, Yang M, Jat P, Li K, Lombardo Y, Xiong D, Coombes RC, Raguz S and Yagüe E: The miR-106b 25 cluster promotes bypass of doxorubicin-induced senescence and increase in motility and invasion by targeting the E-cadherin transcriptional activator EP300. Cell Death Differ 21: 462-474, 2014

35. Sandhu R, Rein J, D'Arcy M, Herschkowitz JI, Hoadley KA and Troester MA: Overexpression of miR-146a in basal-like breast cancer cells confers enhanced tumorigenic potential in association with altered p53 status. Carcinogenesis 35: 2567-2575, 2014.

36. Wang D, Liu D, Gao J, Liu M, Liu S, Jiang M, Liu Y and Zheng D: TRAIL-induced miR-146a expression suppresses CXCR4-mediated human breast cancer migration. FEBS J 280: 3340-3353, 2013 